\title{
Title: Polarimetric receiver in the forty gigahertz band: new instrument for the Q-U-I Joint Tenerife experiment
}

Authors: Enrique Villa ${ }^{1}$, Juan L. Cano ${ }^{1}$, Beatriz Aja ${ }^{1}$, J. Vicente Terán ${ }^{2}$, Luisa de la Fuente ${ }^{1}$, Ángel Mediavilla ${ }^{1}$, Eduardo Artal $^{1}$

Affiliation: ${ }^{1}$ Departamento Ingeniería de Comunicaciones, Universidad de Cantabria, Plaza de la Ciencia s/n, Santander, 39005, Spain

${ }^{2}$ Erzia Technologies, Josefina Sainz de la Maza 4, Santander, 39012, Spain

Corresponding author: villae@unican.es; +34 942200919

ORCID: E. Villa 0000-0003-1028-6957; J. L. Cano 0000-0002-7774-0758; B. Aja 0000-0002-42292334; L. de la Fuente 0000-0003-1403-1660; E. Artal 0000-0002-2569-1894.

\begin{abstract}
This paper describes the analysis, design and characterization of a polarimetric receiver developed for covering the 35 to $47 \mathrm{GHz}$ frequency band in the new instrument aimed at completing the ground-based Q-U-I Joint Tenerife Experiment. This experiment is designed to measure polarization in the Cosmic Microwave Background. The described high frequency instrument is a HEMT-based array composed of 29 pixels. A thorough analysis of the behaviour of the proposed receiver, based on electronic phase switching, is presented for a noise-like linearly polarized input signal, obtaining simultaneously $I, Q$ and $U$ Stokes parameters of the input signal. Wideband subsystems are designed, assembled and characterized for the polarimeter. Their performances are described showing appropriate results within the 35-to-47 GHz frequency band. Functionality tests are performed at room and cryogenic temperatures with adequate results for both temperature conditions, which validate the receiver concept and performance.
\end{abstract}

Keywords: broadband receiver, polarimetry, CMB, astronomical instruments, Stokes parameters, microwave radiometry.

Acknowledgments: The authors would like to thank the Spanish Ministry of Economy, Industry and Competitiveness for the financial support provided through the CONSOLIDER-INGENIO 2010 program under the grant CSD2010-00064 and under the grant ESP2015-70646-C2-2-R. 


\title{
Polarimetric receiver in the forty gigahertz band: new instrument for the Q-U-I Joint Tenerife experiment
}

\author{
Enrique Villa ${ }^{1}$, Juan L. Cano ${ }^{1}$, Beatriz Aja ${ }^{1}$, J. Vicente Terán ${ }^{2}$, Luisa de la Fuente ${ }^{1}$, Ángel \\ Mediavilla ${ }^{1}$, Eduardo Artal $^{1}$ \\ ${ }^{1}$ Departamento Ingeniería de Comunicaciones, Universidad de Cantabria, Plaza de la \\ Ciencia s/n, Santander, 39005, Spain \\ ${ }^{2}$ Erzia Technologies, Josefina Sainz de la Maza 4, Santander, 39012, Spain
}

This paper describes the analysis, design and characterization of a polarimetric receiver developed for covering the 35 to $47 \mathrm{GHz}$ frequency band in the new instrument aimed at completing the ground-based Q-U-I Joint Tenerife Experiment. This experiment is designed to measure polarization in the Cosmic Microwave Background. The described high frequency instrument is a HEMT-based array composed of 29 pixels. A thorough analysis of the behaviour of the proposed receiver, based on electronic phase switching, is presented for a noise-like linearly polarized input signal, obtaining simultaneously $I, Q$ and $U$ Stokes parameters of the input signal. Wideband subsystems are designed, assembled and characterized for the polarimeter. Their performances are described showing appropriate results within the $35-$ to- $47 \mathrm{GHz}$ frequency band. Functionality tests are performed at room and cryogenic temperatures with adequate results for both temperature conditions, which validate the receiver concept and performance.

\section{INTRODUCTION}

The design of wideband and very sensitive receivers is of most importance to radio astronomers since they are key instruments for the study of the relic signals or radiations that help us to explain the origin of the Universe. Among these signals, the Cosmic Microwave Background (CMB) is probably the most important one due to the rich information contained in its intensity and polarization for the explanation of the early Universe. The scientific community has devoted many resources to the study of the CMB through the development of different experiments, such as the Cosmic Background Explorer (COBE) (Richards 2007), the Wilkinson Microwave Anisotropy Probe (WMAP) (Richards 2007) and the PLANCK mission (Aja et al. 2005; Bersanelli et al. 2010; Aghanim et al. 2014). Facing all these satellite experiments, there are many ground-based experiments (National Aeronautics and Space Administration 2017) which have complemented the data obtained from the three spacecraft and enabled to measure other significant CMB parameters. In this way, the Q-U-I Joint Tenerife (QUIJOTE) experiment is aimed at characterizing the polarization of the CMB and other galactic and extragalactic signals at medium and large angular scales in the frequency range from 10 to $47 \mathrm{GHz}$ (Rubiño-Martin et al. 2010; López-Caniego et al. 2014). The configuration of the $40 \mathrm{GHz}$ band receiver (35 to $47 \mathrm{GHz}$ ) is focused on the analysis of the inflationary period of the Universe using CMB data. As a polarimeter, the receiver should enable to obtain $I, Q$ and $U$ Stokes parameters simultaneously and to improve the knowledge of that period of time and complement the data obtained with the Thirty-GHz Instrument (TGI) receiver in the 26 to $36 \mathrm{GHz}$ band (Villa et al. 2015).

This paper presents the analysis, design and characterization of the polarimeter designed for the highest frequency receiver of the QUIJOTE instrument: Forty-GHz Instrument (FGI), verifying its functionality 
according to the theory. A representative polarimeter is analysed obtaining the detected voltages in each output, which depend on the polarization of the incoming signal and the phase state of the receiver. Experimental measurements of the receiver validate the instrument concept comparing measured values with the theoretical ones at room temperature, and, furthermore, a cryogenic cycle is done in order to analyse the behaviour emulating the real operation at the telescope. The document is divided into five sections. The first one gives an introduction whereas the FGI QUIJOTE experiment is described and analysed in section II. Then, in Section III, the different subsystems which form the receiver are explained, giving an overview of their individual performances. Functionality tests, at room and cryogenic temperatures, are presented and discussed in section IV. Finally, section V draws general conclusions.

\section{FGI EXPERIMENT}

The QUIJOTE project is a ground-based experiment installed and operated at El Teide Observatory (Canary Islands, Spain), in which the high frequency receiver, the FGI, is composed of 29 pixels, working in the $35-47 \mathrm{GHz}$ frequency band.

The selected frequency range is due to complement the data obtained with the low frequency receivers and cover all the frequency range just below the inoperative $60-\mathrm{GHz}$ band, which corresponds to the first oxygen absorption line. Moreover, the design of this polarimeter involves a significant reduction in map noise due to the number of receivers whilst a lower synchrotron signal from our galaxy is expected in this frequency band (Rubiño-Martin et al. 2010; López-Caniego et al. 2014).

The proposed receiver configuration improves the performance of the TGI one (Villa et al. 2015; Villa 2014), since a higher integration level and isolation performance have been achieved. The new topology of the correlation and detection module regarding the TGI receiver provides improved microwave results in terms of reflection coefficients and isolation between branches. Besides, a more accurate phase control of broadband hybrids in order to correlate the signal of receiver branches is achieved, which implies in a better agreement between the measured output values and the theoretical expected ones.

\subsection{FGI Receiver Block Diagram}

As in the QUIJOTE TGI receiver (Villa et al. 2015; Villa 2014), the FGI polarimeter is intended to measure three of the Stokes parameters ( $I, Q$ and $U$ ) simultaneously in order to characterize the polarization of the CMB. These parameters are obtained after combining measurable output signals in the receiver.

The block diagram of each pixel in the FGI polarimeter is shown in Fig. 1, which is divided into a cold stage module (20 K) and a room temperature (298 K) part. The cryogenic part is made up of a feedhorn antenna, a polarizer, an orthomode transducer (OMT) and two low-noise amplifiers. This part provides two output signals at the OMT, proportional to left- and right-hand circular polarization components of the wave received by the antenna. These signals are amplified by two identical cryogenic low-noise amplifiers and, then, combined and processed in the Back-End Module of the receiver. Hence, outside the cryostat, further amplification, filtering, electrical phase switching and correlation and detection stages are performed at room temperature. Finally, the microwave signals are converted into DC voltages which are processed by a data acquisition system (DAS).

The polarimeter operation is based on electronic phase switching, with sixteen phase states generated by individual phase shifts of $0^{\circ}, 90^{\circ}, 180^{\circ}$ or $270^{\circ}$ in each receiver branch (Villa et al. 2016), which provides four equivalent phase states in the receiver, each one obtained with four different combinations of the phase switches. 


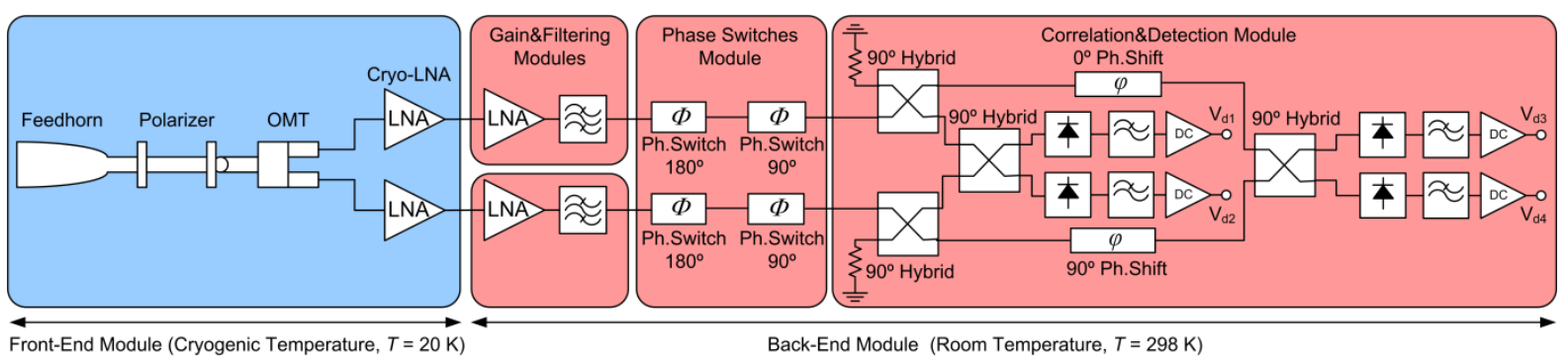

Fig. 1 Block diagram of a single pixel of the FGI QUIJOTE receiver

\subsection{FGI Receiver Analysis}

The analysis of the receiver consists in expounding the relationship between the four detected voltages of the system and the Stokes parameters and on determining the total system noise temperature based on all the noise contributions in a ground-based receiver.

\subsubsection{Stokes parameters calculation}

In each pixel, a set of four outputs voltages ( $V_{d 1}$ to $\left.V_{d 4}\right)$ are expected as shown in Fig. 1, providing detected DC levels. The combination of the polarizer and the OMT in the Front-End Module of the receiver separates the incoming linearly polarized signal to circular components by rotating $45^{\circ}$ the reference system of the polarizer regarding the OMT.

As stated before, the definition of the polarimeter enables the measurement of the $I, Q$ and $U$ Stokes parameters (Collet 2005), which are expressed in a circular coordinate system as

$$
\begin{gathered}
I=\left|E_{l}\right|^{2}+\left|E_{r}\right|^{2} \\
Q=2 \cdot \operatorname{Re}\left(E_{l}^{*} \cdot E_{r}\right) \\
U=-2 \cdot \operatorname{Im}\left(E_{l}^{*} \cdot E_{r}\right)
\end{gathered}
$$

where $E_{l}$ and $E_{r}$ are the left and right circular polarization electrical field components, respectively. To simplify the notation, $V_{L}$ and $V_{R}$ are defined as the signals proportional to the left-hand and right-hand circular electric field components at the OMT output, respectively.

The analysis of the receiver operation depends on the phase state of the Phase Switches Module. To simplify it, a reduced diagram of a FGI pixel is considered and shown in Fig. 2. Each phase state $\Phi_{T}$ can be defined in terms of the phase difference between branches of the pixel as

$$
\Phi_{T}=\Phi_{\mathrm{B} 2}-\Phi_{\mathrm{B} 1}=\left(\Phi_{3}+\Phi_{4}\right)-\left(\Phi_{1}+\Phi_{2}\right)
$$

where $\Phi_{\mathrm{B} 2}$ corresponds to the insertion phase of the lower branch of the Phase Switch Module and $\Phi_{\mathrm{B} 1}$ to the insertion phase of the upper branch of the Phase Switches Module in Fig. 2.

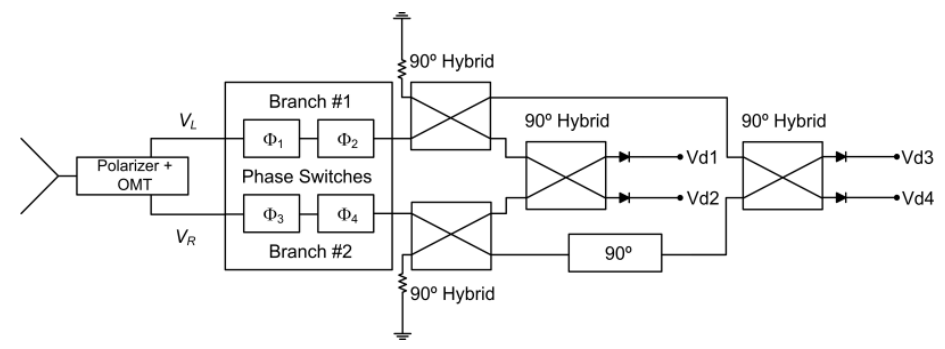

Fig. 2 Simplified diagram of a single FGI pixel 
As initial step, the reference phase state is assumed in the Phase Switches Module, in which both branches are configured in the $0^{\circ}$ phase state. Hence, the detected output voltages are obtained as

$$
\begin{aligned}
& V_{d 1} \propto\left|V_{L}-j \cdot V_{R}\right|^{2} \\
& V_{d 2} \propto\left|V_{L}+j \cdot V_{R}\right|^{2} \\
& V_{d 3} \propto\left|V_{L}-V_{R}\right|^{2} \\
& V_{d 4} \propto\left|V_{L}+V_{R}\right|^{2}
\end{aligned}
$$

In the particular analysis of a linearly polarized signal, two $90^{\circ}$ out-of-phase signals are expected at the OMT outputs when a linearly polarized signal incomes the system. Therefore, the signals proportional to each circular polarization components accomplish that $V_{R}=j \cdot V_{L}$. Then, the Stokes parameters are obtained by performing linear combination between the output values, and the following combinations are achieved:

$$
\begin{gathered}
V_{d 1}+V_{d 2} \propto\left|V_{L}+V_{L}\right|^{2}+\left|V_{L}-V_{L}\right|^{2} \propto I \\
V_{d 3}+V_{d 4} \propto\left|V_{L}-j \cdot V_{L}\right|^{2}+\left|V_{L}+j \cdot V_{L}\right|^{2} \propto I \\
V_{d 1}-V_{d 2} \propto\left|V_{L}+V_{L}\right|^{2}-\left|V_{L}-V_{L}\right|^{2} \propto Q \\
V_{d 3}-V_{d 4} \propto\left|V_{L}-j \cdot V_{L}\right|^{2}-\left|V_{L}+j \cdot V_{L}\right|^{2} \propto U
\end{gathered}
$$

Similarly, the Stokes parameters are calculated for the other phase states by means of the modification of the value of $\Phi_{T}$, and their values are listed in Table 1 . Voltage values are used to calculate the values of the Stokes parameters, which are normalized by its maximum value, corresponding to the intensity parameter $I$, to fulfil their theoretical range, with maximum $I, Q$ and $U$ amplitudes equal to the unity.

Table 1 Stokes parameters from output detected voltages

\begin{tabular}{cccc}
\hline \hline$\Phi_{\mathrm{T}}$ & $I$ & $Q$ & $U$ \\
\hline $0^{\circ}$ & $V_{d 1}+V_{d 2}=V_{d 3}+V_{d 4}$ & $V_{d 2}-V_{d 1}$ & $V_{d 4}-V_{d 3}$ \\
$90^{\circ}$ & $V_{d 1}+V_{d 2}=V_{d 3}+V_{d 4}$ & $V_{d 4}-V_{d 3}$ & $V_{d 2}-V_{d 1}$ \\
$180^{\circ}$ & $V_{d 1}+V_{d 2}=V_{d 3}+V_{d 4}$ & $V_{d 2}-V_{d 1}$ & $V_{d 4}-V_{d 3}$ \\
$270^{\circ}$ & $V_{d 1}+V_{d 2}=V_{d 3}+V_{d 4}$ & $V_{d 4}-V_{d 3}$ & $V_{d 2}-V_{d 1}$ \\
\hline \hline
\end{tabular}

The CMB is expected to be linearly polarized, therefore, the analysis of the polarimeter is focused when a linearly polarized input signal is coming to the receiver. Hence, for an $\mathrm{x}$-axis linearly polarized input signal, the detected outputs according to (5)-(8) for the phase state $\Phi_{T}=0$ are expressed as

$$
\begin{gathered}
V_{d 1}=K \cdot\left|V_{L}\right|^{2} \\
V_{d 2}=0 \\
V_{d 3}=\frac{K}{2} \cdot\left|V_{L}\right|^{2} \\
V_{d 4}=\frac{K}{2} \cdot\left|V_{L}\right|^{2}
\end{gathered}
$$

where $K$ is a constant related to the full responsivity of the receiver chain. By changing the phase state of the phase switches module, the output voltage values for the different phase states are obtained and listed in Table 2.

Table 2 Detected voltages for an x-axis linearly polarized signal 


\begin{tabular}{ccccc}
\hline \hline$\Phi_{T}$ & $V_{d 1}$ & $V_{d 2}$ & $V_{d 3}$ & $V_{d 4}$ \\
\hline $0^{\circ}$ & $K \cdot\left|V_{L}\right|^{2}$ & 0 & $(K / 2) \cdot\left|V_{L}\right|^{2}$ & $(K / 2) \cdot\left|V_{L}\right|^{2}$ \\
$90^{\circ}$ & $(K / 2) \cdot\left|V_{L}\right|^{2}$ & $(K / 2) \cdot\left|V_{L}\right|^{2}$ & $K \cdot V_{L}{ }^{2}$ & 0 \\
$180^{\circ}$ & 0 & $K \cdot\left|V_{L}\right|^{2}$ & $(K / 2) \cdot\left|V_{L}\right|^{2}$ & $(K / 2) \cdot\left|V_{L}\right|^{2}$ \\
$270^{\circ}$ & $(K / 2) \cdot\left|V_{L}\right|^{2}$ & $(K / 2) \cdot\left|V_{L}\right|^{2}$ & 0 & $K \cdot\left|V_{L}\right|^{2}$ \\
\hline \hline
\end{tabular}

\subsubsection{Noise Analysis}

The previous analysis is complemented with the evaluation of the noise temperature of the receiver. As a ground-based experiment, the total system noise temperature is not only affected by the involved subsystems in the polarimeter, but also influenced by the atmosphere. Then, the system noise temperature $T_{\text {sys }}$ (Otoshi 2008) can be expressed as

$$
T_{\text {sys }}=T_{\text {atm }}+\frac{T_{C M B}}{L_{a t m}}+T_{\text {receiver }}
$$

where $T_{a t m}$ is the effective atmosphere temperature, $T_{C M B}$ is the CMB temperature, $L_{a t m}$ is the attenuation of the atmosphere and $T_{\text {receiver }}$ is the noise contribution of the FGI receiver. This contribution is given by

$$
T_{\text {receiver }}=T_{\text {antenna }}+T_{\text {spillover }}+T_{r}
$$

where $T_{\text {antenna }}$ is the equivalent antenna noise temperature, $T_{\text {spillover }}$ is the effective noise temperature added by the fraction of power received which originates from the ground, and the receiver noise temperature $T_{r}$ is

$$
T_{r}=\left(L_{\text {feed }}-1\right) \cdot T_{p}+L_{\text {feed }} \cdot T_{r}^{\prime}=\left(L_{\text {feed }}-1\right) \cdot T_{p}+L_{\text {feed }} \cdot\left(T_{L N A}+\frac{\left(L_{c}-1\right)}{G_{L N A}} \cdot T_{a}+\frac{L_{c}}{G_{L N A}} \cdot T_{B E M}\right)
$$

where $T_{p}$ is the cryogenic physical temperature, $L_{\text {feed }}$ is the insertion loss of the polarizer and the OMT, $T_{L N A}$ is the equivalent noise temperature of the cryogenic LNA, $T_{a}$ is the physical room temperature, $L_{c}$ is the insertion loss of the interconnection element between the cryogenic and the room temperature parts, $G_{L N A}$ is the gain of the cryogenic LNA and $T_{B E M}$ is the noise temperature of the Back-End Module. Fig. 3 shows a block diagram of this system.

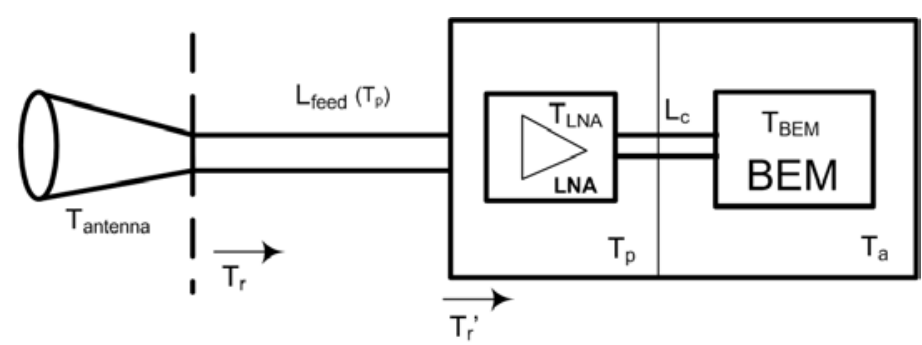

Fig. 3 Block diagram of the system for the noise analysis

\section{FGI POLARIMETER SUBSYSTEMS}

The different subsystems that are part of the FGI receiver, from the cryogenic part to the room temperature one, have been individually assembled and experimentally characterized.

The feed subsystems are waveguide components, such as the feedhorn antenna, the polarizer and the OMT, which are tested at room temperature, since only a reduction on their losses are expected when they operate under cryogenic conditions. The feedhorn antenna is based on a corrugated design (Cano et al. 2011) using a circular waveguide input. The cross-polarization of the antenna is a significant issue, and it is defined by 
the throat of the antenna. A view of its measurement process is shown in Fig. 4 and its results are depicted in Fig. 5, in terms of the directivity, input return loss, worst value of cross-polarization for $\mathrm{Phi}=45^{\circ}$ and radiation patterns at $41 \mathrm{GHz}$ (co-polar at $\mathrm{Phi}=0^{\circ}$ and $\mathrm{Phi}=90^{\circ}$, and cross-polar at $\mathrm{Phi}=45^{\circ}$ ).

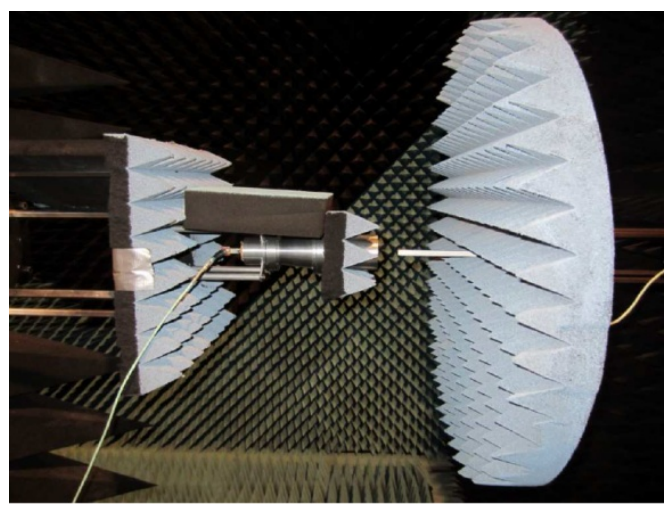

Fig. 4 Detail of the measurement of the FGI feedhorn antenna in an anechoic chamber

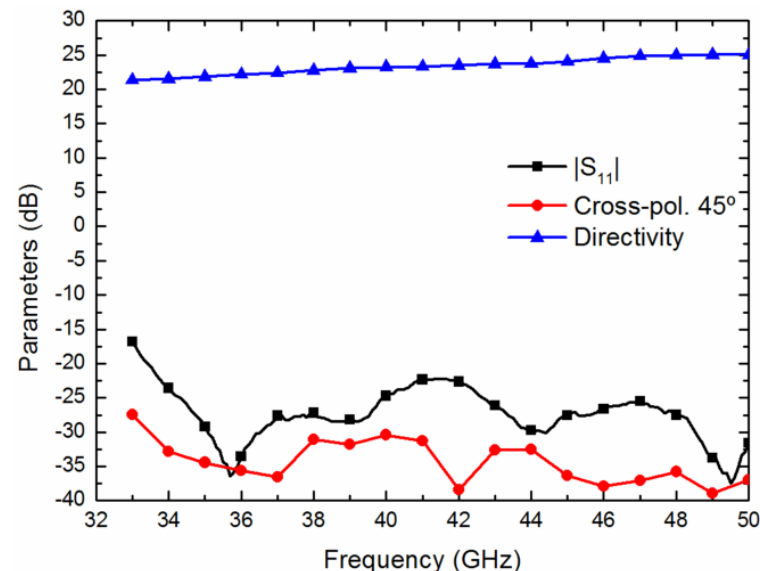

(a)

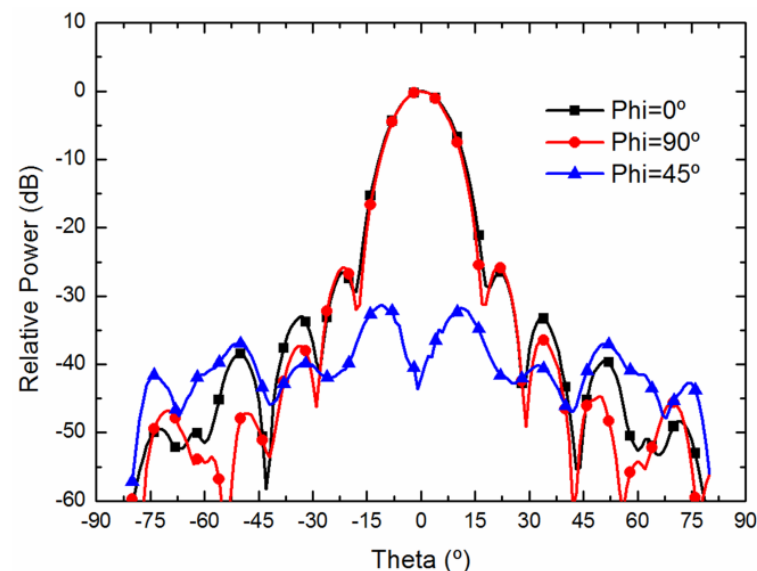

(b)

Fig. 5 FGI feedhorn antenna results. (a) Input matching, cross-polarization and directivity. (b) Radiation pattern at $41 \mathrm{GHz}$

The polarizer is a square waveguide component (Tribak et al. 2009) with stepped ridges in the four internal walls of the waveguide. The ridges are carefully designed to achieve an accurate wideband $90^{\circ}$ phase difference between $\mathrm{TE}_{10}$ and $\mathrm{TE}_{01}$ orthogonal propagated modes in a square waveguide. Some views of a manufactured polarizer are shown in Fig. 6, while measured results are depicted in Fig. 7.
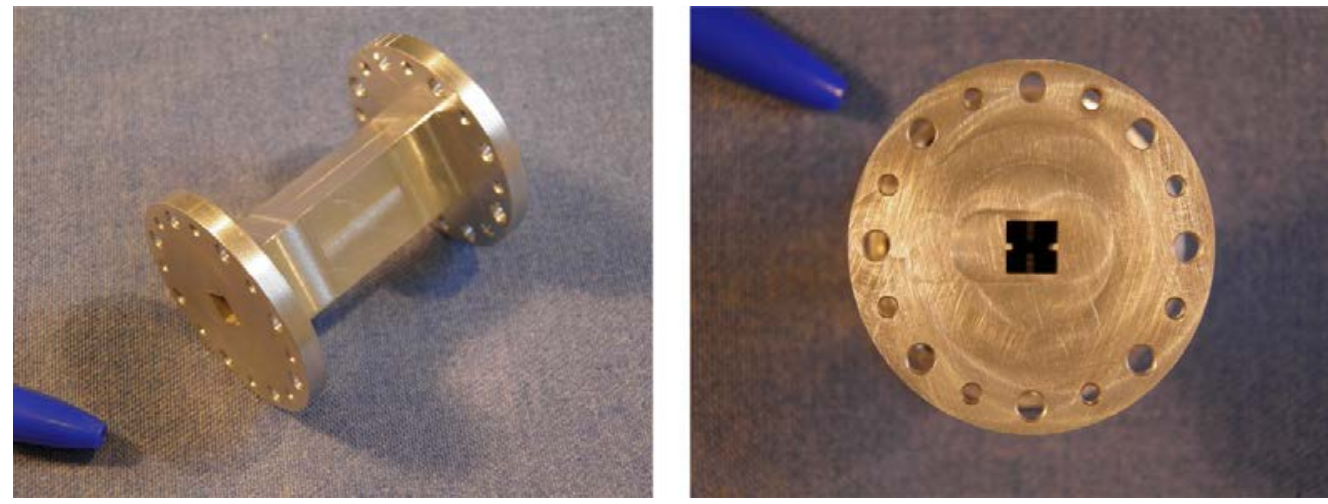

Fig. 6 FGI polarizer views 


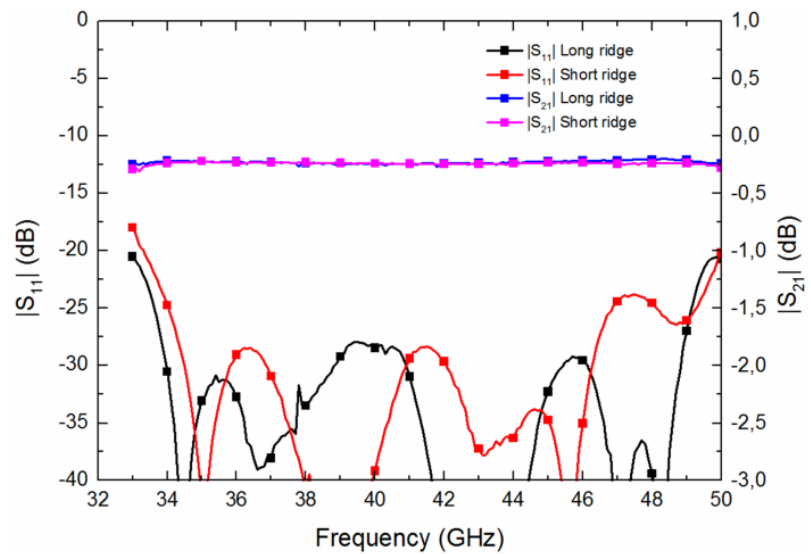

(a)

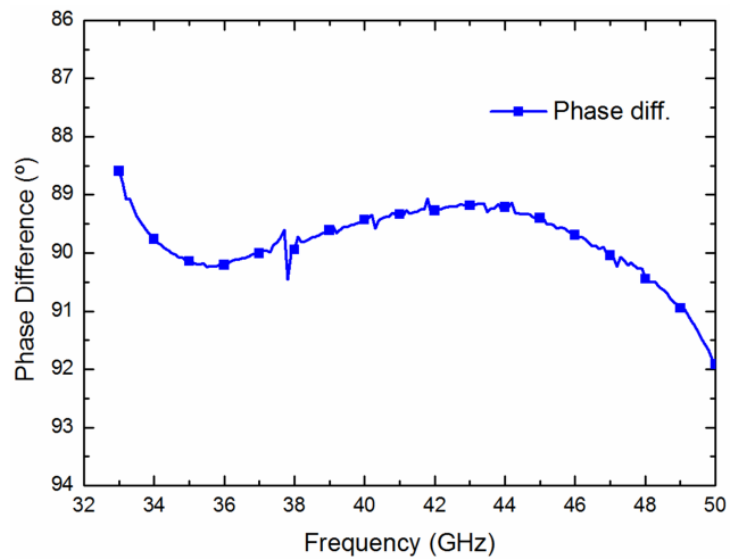

(b)

Fig. 7 FGI polarizer results. (a) Input matching and insertion loss for both ridge sections. (b). Phase difference between the two propagated modes

The OMT (Cano et al. 2010) is the device which enables the component separation of the incoming signal. It is based on a turnstile junction with an inserted scatterer. A view of the OMT is shown in Fig. 8. It is designed with a circular input waveguide and two WR22 rectangular waveguide in-phase outputs. The output reflection coefficients in WR22 waveguide are measured by connecting a circular radiating load at the input, as well as the isolation between them as the transmission between both accesses. To characterize the transmission losses between the circular port and each rectangular one, a short-circuit is placed in the circular port and they are estimated as one half of the measured reflection coefficient in each port, since the isolation results between rectangular ports is very high. The measured results, in terms of the insertion loss, matching in rectangular ports, isolation and phase difference between transmission paths, are shown in Fig. 9.

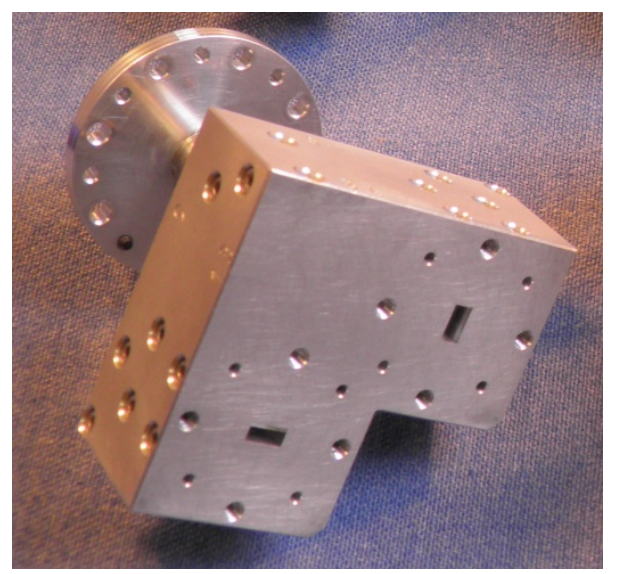

Fig. 8 FGI OMT photograph 


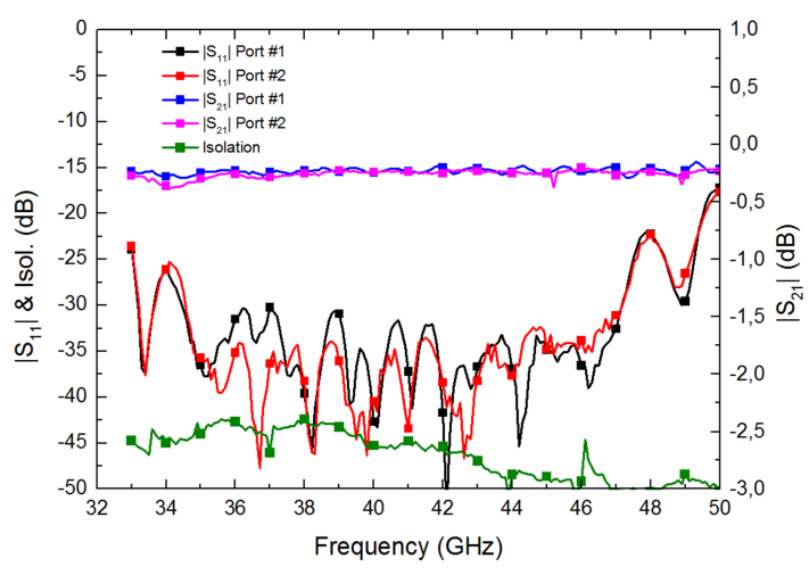

(a)

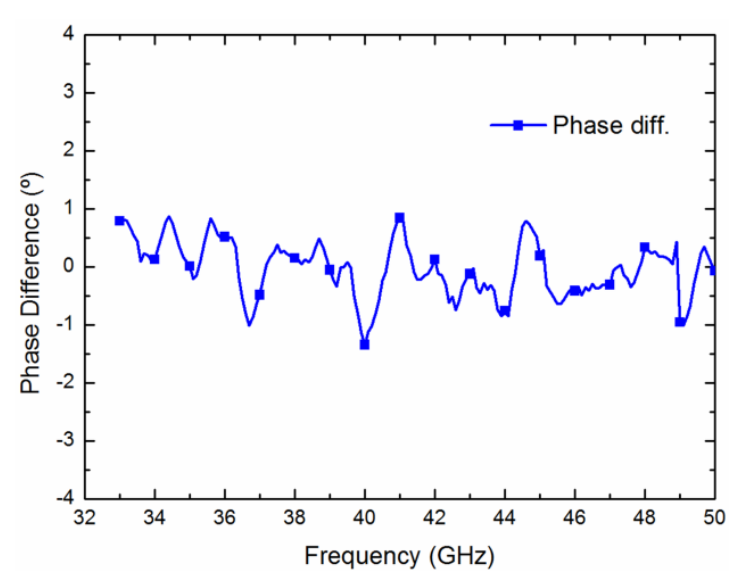

(b)

Fig. 9 FGI OMT measurements. (a) Rectangular waveguide reflection coefficients, transmission losses and isolation between outputs. (b) Phase difference between both transmission paths

The cryogenic low-noise amplifier (LNA) is composed of two cascaded identical monolithic microwave integrated circuit (MMIC) LNAs with an equalizer in-between. The MMIC LNA design is based on 100-nm metamorphic high-electron-mobility-transistor (mHEMT) technology from the Fraunhofer IAF (Freiburg - Germany), which shows outstanding performance operating at cryogenic temperatures (Aja et al. 2012). In order to compensate the negative gain slope they exhibit, a microstrip equalizer based on Zhou et al. (2012) is designed and placed between them. The resistors of the equalizer are directly etched over the alumina substrate using a $20 \Omega$ /square resistive layer. An internal view of a cryogenic LNA is shown in Fig. 10 . The test of the device is performed when it is cooled down to $15 \mathrm{~K}$, showing an average insertion gain of $49.8 \mathrm{~dB}$ and noise temperature of $15.9 \mathrm{~K}$ within the 35-to-47 GHz band with a total power consumption of $27.2 \mathrm{~mW}$ (Terán 2017). The results are shown in Fig. 11.

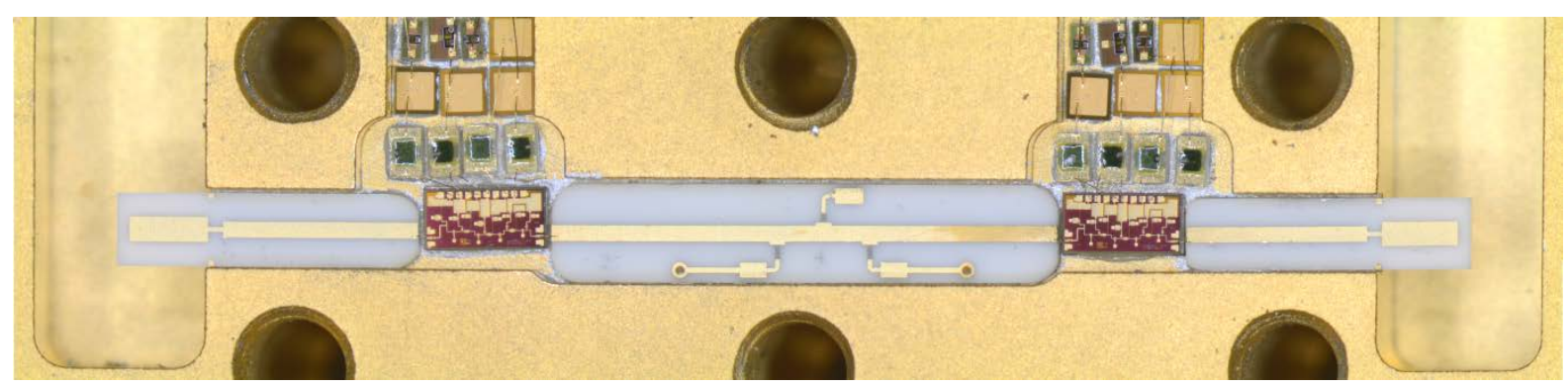

Fig. 10 FGI cryogenic low-noise amplifier internal view 


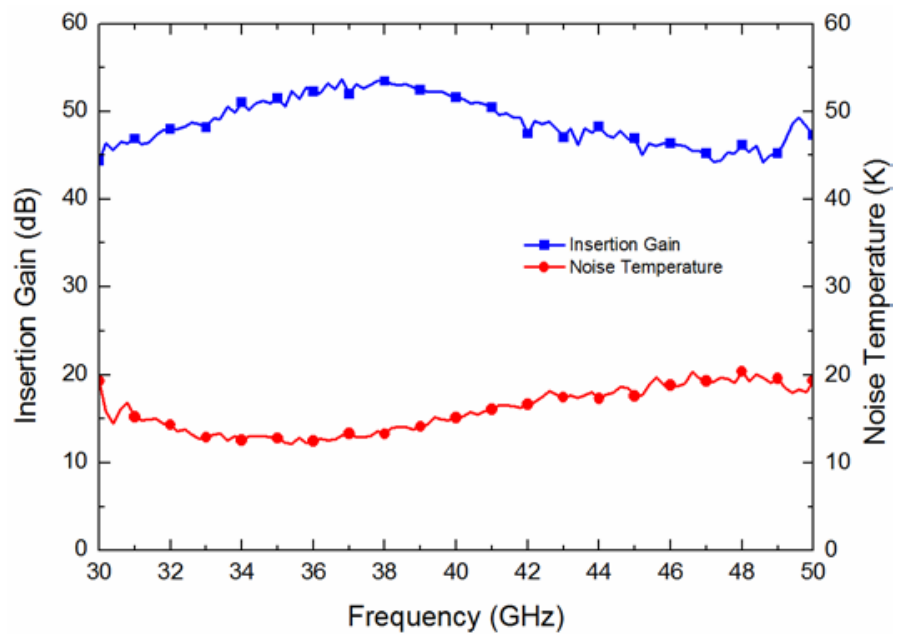

Fig. 11 FGI cryogenic low-noise amplifier insertion gain and noise temperature

The room temperature subsystems for the FGI polarimeter include further amplification, phase switching and correlation and detection. The first module is the Gain\&Filtering Module depicted in Fig. 1. It is composed of two cascaded monolithic commercial LNAs with an equalizer in-between and a microstrip filter to define the band. The first LNA used in the module is a four-stage amplifier model CGY2122XUH from OMMIC, while the second one is the model HMC1126 from Hittite. The equalization of the microwave response is accomplished using in-house microstrip design (Terán 2017) based on the use of series and shunt etched resistors over an alumina substrate with a $50 \Omega$ /square resistive layer. Finally, the microstrip filter, also designed on alumina substrate, confines the 35-to-47 GHz frequency band. An internal view of the module is shown in Fig. 12 and its performance, in terms of gain and matching for both rectangular ports, is shown in Fig. 13.

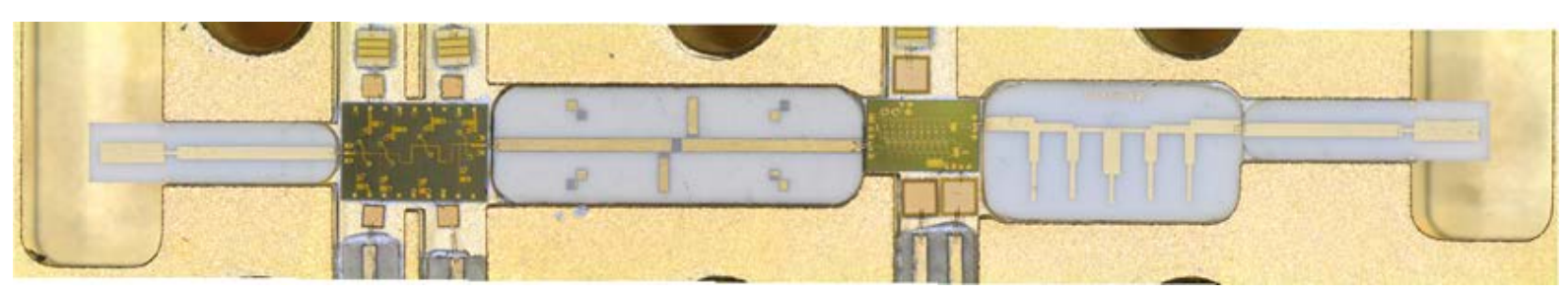

Fig 12 FGI gain and filtering module internal view

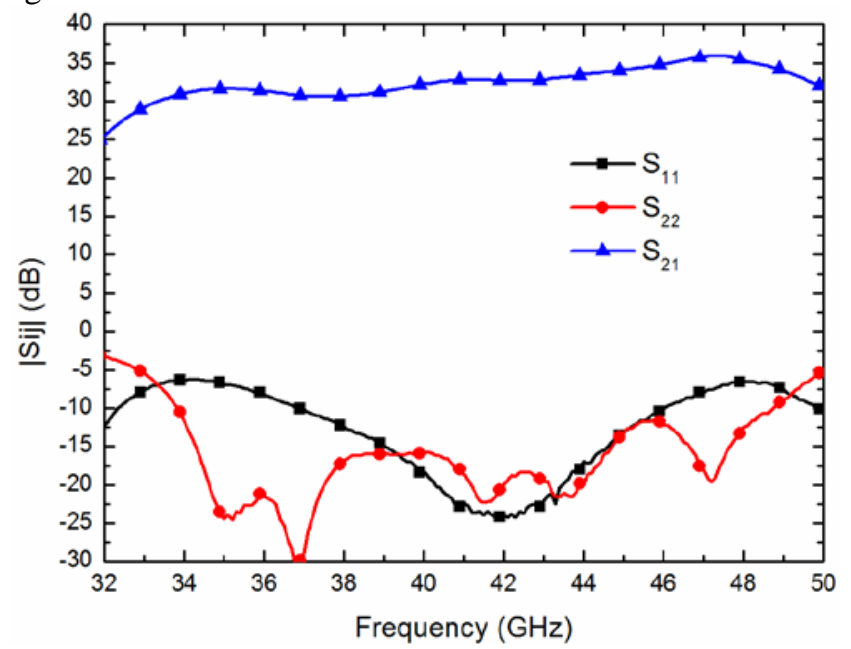

Fig. 13 FGI gain and filtering module measurement: input and output return loss and gain 
The Phase Switches Module is a WR22 waveguide chassis based on a four state phase shifter with PIN diodes as switching devices for each receiver branch. The microstrip circuit has two phase shifting branches with a $90^{\circ}$ fixed phase difference which are combined using Wilkinson dividers, and each branch has an individual $0 / 180^{\circ}$ phase shifter. Further details are described by Villa et al. (2016). Besides, the module provides full switching functionality with the use of transistor-transistor logic (TTL) drivers, model DR65-0109 from MACOM Technology. A detailed view of the assembly inside the chassis is shown in Fig. 14. The phase performance of the module shows average phase shifts of $88.5^{\circ}, 178.3^{\circ}$ and $269.3^{\circ}$ over the band (Villa et al. 2016).

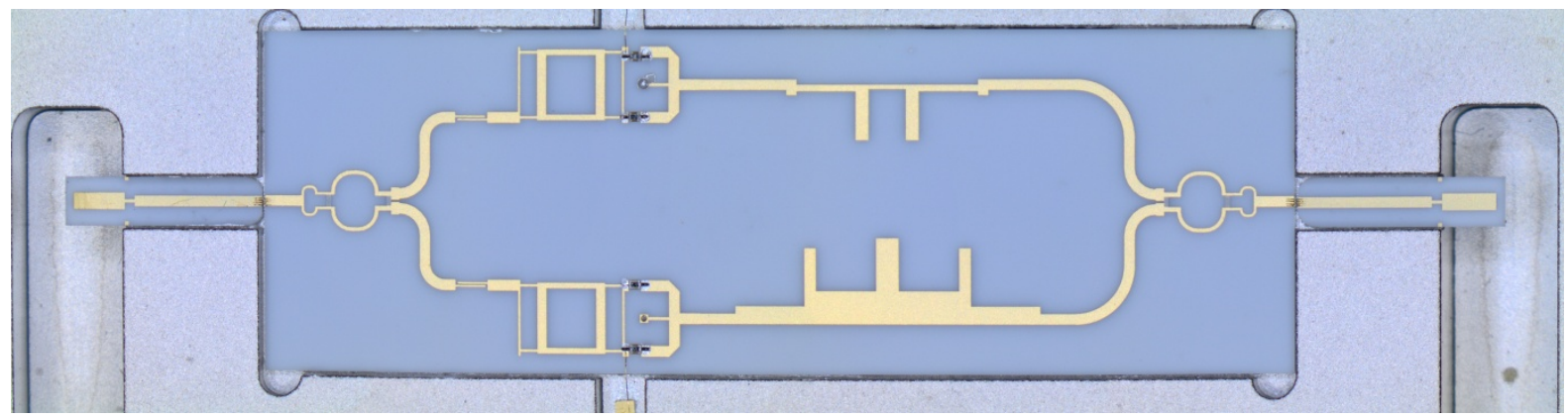

Fig. 14 FGI phase switches module internal view

Finally, the Correlation and Detection Module has WR22 waveguide input and output ports and includes $90^{\circ}$ waveguide hybrids, a fixed $90^{\circ}$ waveguide phase shifter and Schottky diode detectors. The waveguide hybrids provide high isolation values and accurate $90^{\circ}$ phase shifts between their ports, which involves a better precision in the power division of the microwave signal. A set of four hybrids couplers are involved. The inputs couplers separate the input signals with same magnitude and $90^{\circ}$ out of phase, whilst the other pair of hybrid couplers in the module produce the correlation operations, addition and subtraction, between the input signals. The remaining port in each hybrid coupler is terminated in a matched load manufactured with absorber material Eccosorb MF124. Besides, the fixed $90^{\circ}$ waveguide phase shifter shows an extremely low inband phase error, so the phase of the incoming signals to the detector is highly controlled. These features cause that the mid-voltage output signals for each state show balanced levels. Finally, microstrip detectors (Villa 2014) based on the Schottky diode HSCH-9161 from Agilent Technologies convert microwave signals to DC output voltages which are amplified by operational amplifiers. An internal view of the module is shown in Fig. 15, while experimental tests are shown in Fig. 16 for $90^{\circ}$ out-of-phase input signals to both waveguide input ports: the first test is a frequency sweep using a constant input power of $-20 \mathrm{dBm}$, and the second test is a power sweep for a fixed frequency of $41 \mathrm{GHz}$. 


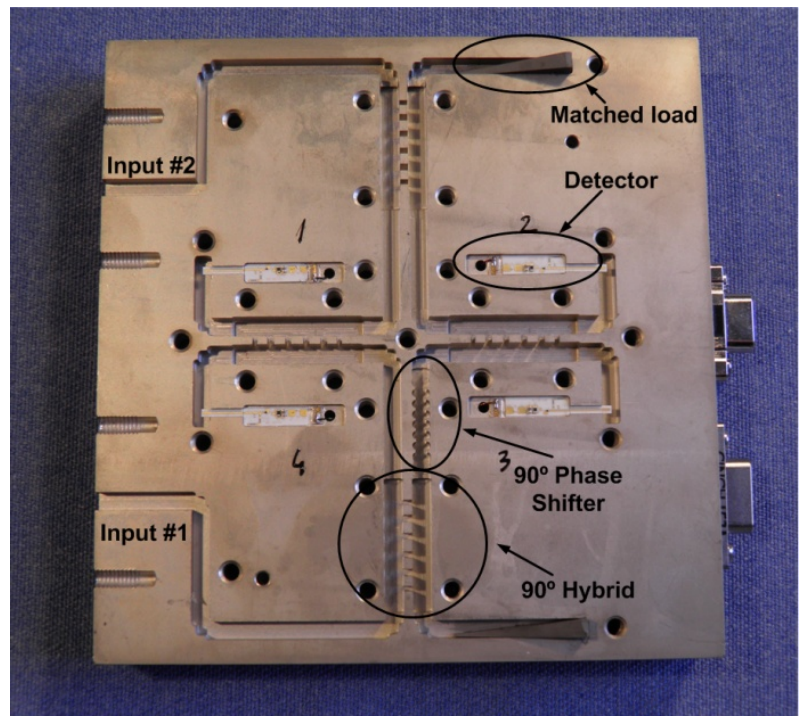

Fig. 15 FGI detection and correlation module internal view

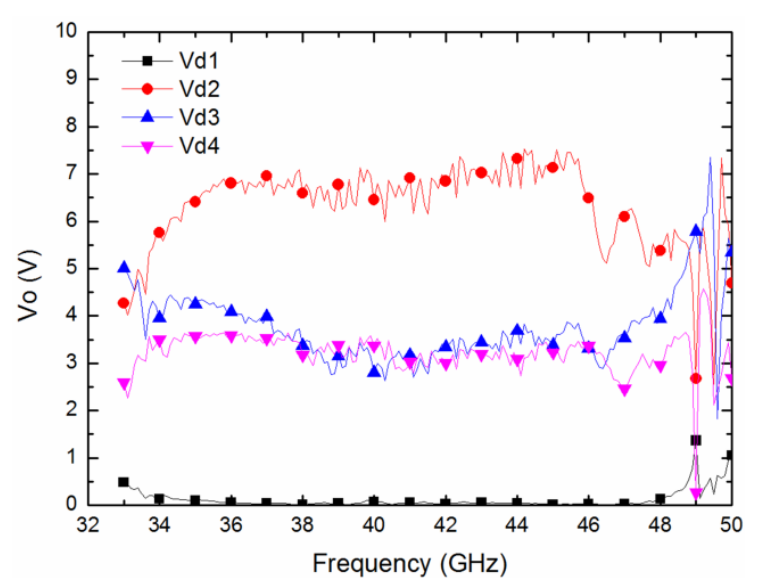

(a)

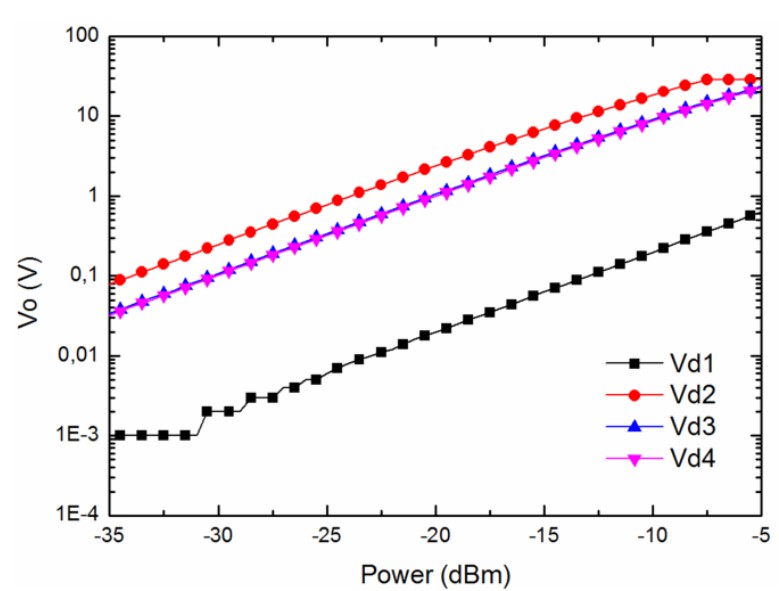

(b)

Fig. 16 Output voltages from the FGI detection and correlation module. (a) Frequency sweep for an input power of $-20 \mathrm{dBm}$. (b) Power sweep for an input frequency of $41 \mathrm{GHz}$

\section{FGI POLARIMETER TEST}

Once all the subsystems have been described, their joint operation is presented. Their functionality as a polarimeter is focused on two different tests. First, a room temperature characterization in order to validate the theory behind the receiver. Then, a cryogenic test in which its real operation is analysed.

\subsection{Room Temperature Performance}

The room temperature functionality test of a FGI polarimeter pixel consists of providing a broadband linearly polarized signal at the receiver input (feedhorn) and acquiring the detected voltages at the Correlation and Detection Module outputs. A specific board is designed including all the Back-End subsystems and interconnections, which is depicted in Fig. 17, in order to provide a robust configuration for each pixel. The configuration shown in the picture causes an additional $180^{\circ}$ phase shift between both input branches, since the Gain and Filtering Modules have to be assembled in opposite arrangement by mechanical constraints. 


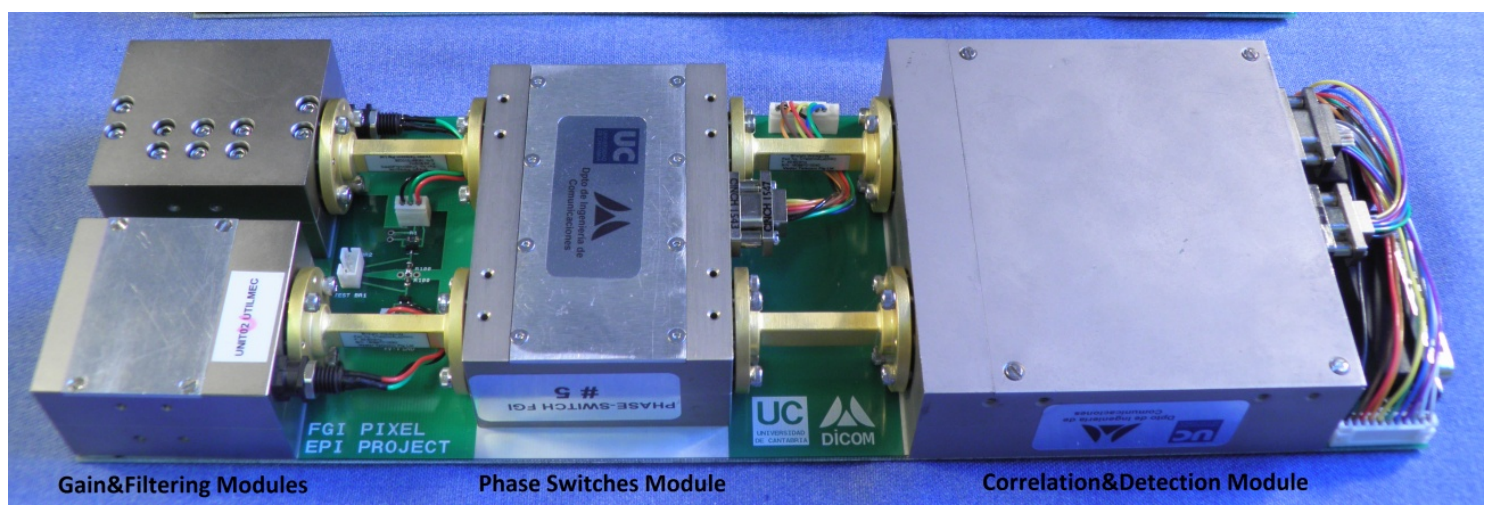

Fig. 17 Back-End Module board including the assembly of the subsystems

The test includes all the Front-End Module passive components working at room temperature, while the cryogenic LNAs are not included in order to avoid saturation on the receiver due to an excessive gain and noise levels.

A broadband noise-like $\mathrm{x}$-axis (horizontal) linearly polarized signal is used to excite the pixel, accomplished with a noise source model 346CK01 from Agilent Technologies and, then, amplified (around $33 \mathrm{~dB}$ ) and transmitted by a conical horn with rectangular waveguide input, which defines the polarization axis. The conical transmitting horn and the feedhorn antenna of the pixel are separated a fixed distance, which is enough to avoid receiver gain compression and ensure linear operation. Additional waveguide to 2.4 mm coaxial transitions are used in order to connect the OMT outputs to the BEM input using flexible cables. Besides, the individual pixel board is assembled inside a rack, which contains 10 units. The test bench for this test is shown in Fig. 18.

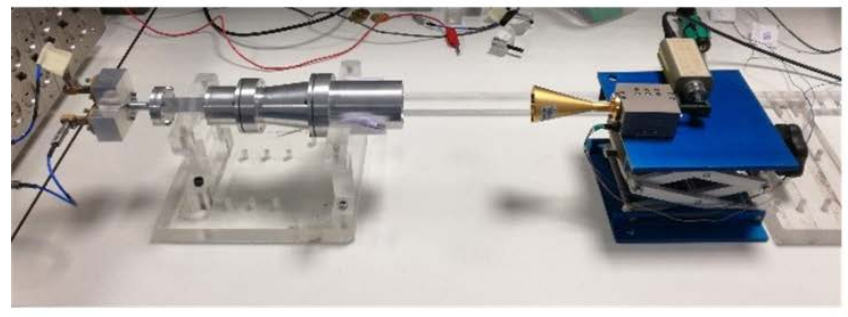

(a)

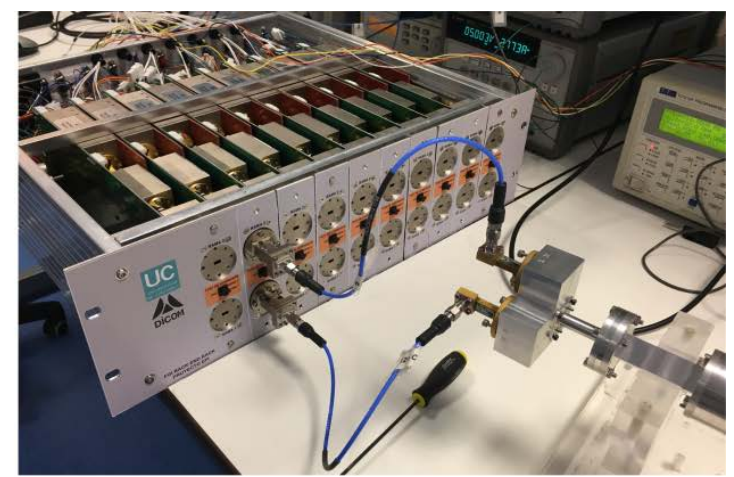

(b)

Fig. 18 Pictures of the polarimeter test bench. (a) Front-End feed system on the left receives an x-axis linearly polarized signal from the conical feedhorn on the right. (b) Connection from OMT outputs to a Back-End Module pixel input by two phase balanced coaxial cables

The test consists of measuring the detected voltages of the receiver in a defined period of time in which all the phase states are swept. Therefore, a set of TTL signals controls the Phase Switches Module in order to change their state. The system is configured with a sampling rate of $1 \mathrm{kHz}$, acquiring one second of data using a PXI system. The output voltages of a pixel are measured and their values are shown in Fig. 19. 


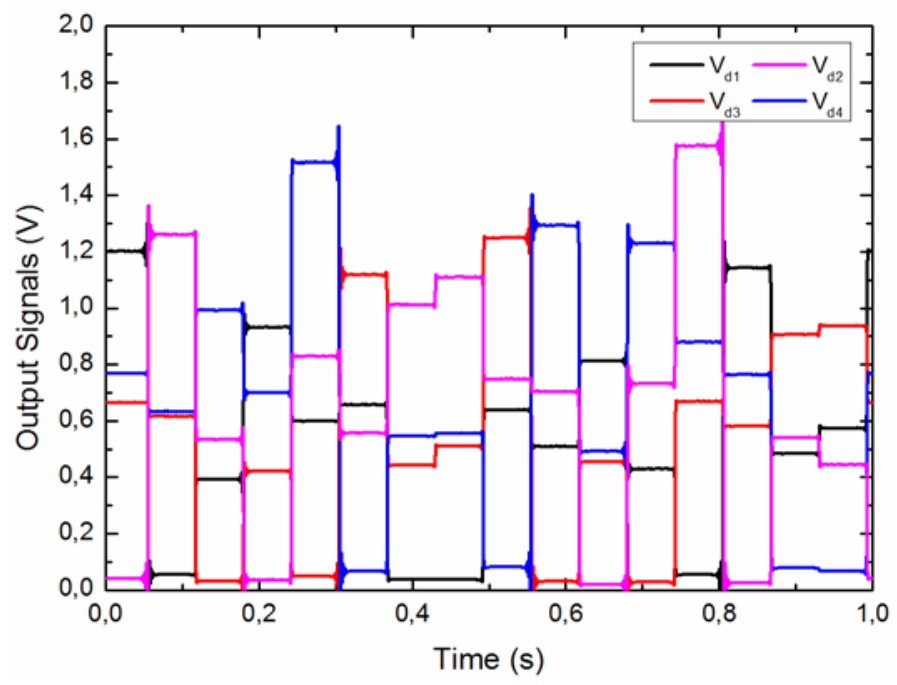

Fig. 19 Output signals of a pixel inside the BEM rack for an X-axis linear polarization input signal

The average values of the four detected voltages $\left(V_{d 1}\right.$ to $\left.V_{d 4}\right)$ for each phase state are listed in Table 3, as well as the values of the $Q$ and $U$ parameters for $I=1$. The listed values corresponds to the order expected in the receiver according to the TTL control signals, in which the so-called state ' 0 ', corresponding to a low level signal in the TTL control signals, occurs when the maximum voltage in $V_{d 2}$ output is measured in two consecutive states.

Table 3 Average detected voltages in each output, and $Q$ and $U$ Stokes parameters for each phase state for $I=1$

\begin{tabular}{cccccccc}
\hline State & $\Phi_{T}\left(^{\circ}\right)$ & $V_{d 1}(V)$ & $V_{d 2}(V)$ & $V_{d 3}(V)$ & $V_{d 4}(V)$ & $Q$ & $U$ \\
\hline 0 & 0 & 0.03 & 1.11 & 0.51 & 0.55 & 0.98 & 0.04 \\
1 & 270 & 0.64 & 0.75 & 1.25 & 0.08 & 0.86 & 0.08 \\
2 & 90 & 0.51 & 0.7 & 0.03 & 1.29 & 0.99 & 0.15 \\
3 & 180 & 0.81 & 0.02 & 0.45 & 0.49 & 0.89 & 0.05 \\
4 & 90 & 0.44 & 0.73 & 0.03 & 1.23 & 0.99 & 0.24 \\
5 & 0 & 0.05 & 1.57 & 0.67 & 0.88 & 0.96 & 0.13 \\
6 & 180 & 1.14 & 0.03 & 0.58 & 0.76 & 0.88 & 0.14 \\
7 & 270 & 0.48 & 0.54 & 0.91 & 0.07 & 0.84 & 0.06 \\
8 & 270 & 0.57 & 0.44 & 0.93 & 0.06 & 0.87 & 0.13 \\
9 & 180 & 1.2 & 0.04 & 0.66 & 0.76 & 0.87 & 0.08 \\
10 & 0 & 0.05 & 1.26 & 0.62 & 0.63 & 0.95 & 0.01 \\
11 & 90 & 0.39 & 0.53 & 0.03 & 0.99 & 0.99 & 0.14 \\
12 & 180 & 0.93 & 0.03 & 0.42 & 0.7 & 0.87 & 0.27 \\
13 & 90 & 0.59 & 0.83 & 0.04 & 1.51 & 0.99 & 0.16 \\
14 & 270 & 0.65 & 0.55 & 1.12 & 0.07 & 0.88 & 0.08 \\
15 & 0 & 0.03 & 1.01 & 0.44 & 0.54 & 0.97 & 0.10 \\
\hline \hline
\end{tabular}

\subsection{Cryogenic Temperature Performance}

The cryogenic test of the FGI polarimeter pixel is performed with the Front-End and the Back-End Modules working at $15 \mathrm{~K}$ and $300 \mathrm{~K}$ ambient temperatures respectively. A simplified schematic of the configured set-up with the parts of the receiver involved is shown in Fig. 20. The Front-End Module for this test 
consists of a WR22 waveguide termination connected through a stainless steel (SS) waveguide to a $3 \mathrm{~dB}$ inphase waveguide power divider and a pair of cryogenic LNAs inside a cryostat. Consequently, the feedhorn antenna, the polarizer and the OMT are replaced with the first three previously listed elements. A picture of the set-up inside the cryostat is shown in Fig. 21. The set-up is provided with temperature sensors located on the cryogenic LNAs and on the waveguide termination to control the working temperatures inside the cryostat. In addition, a heater is attached to the waveguide termination, which allows to introduce a range of input noise temperatures to the receiver by modifying the physical temperature of the termination. Then, the waveguide power divider distributes the input noise from the waveguide termination, and its outputs are connected to both cryogenic LNAs without any phase difference between them.

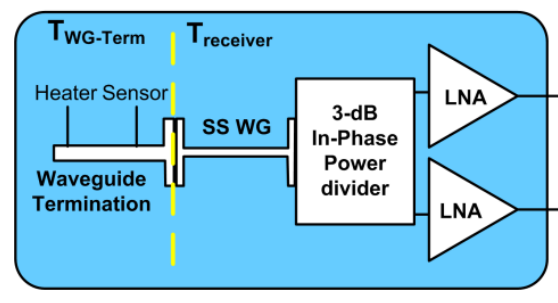

Front-End Module $(\mathrm{T}=15 \mathrm{~K})$

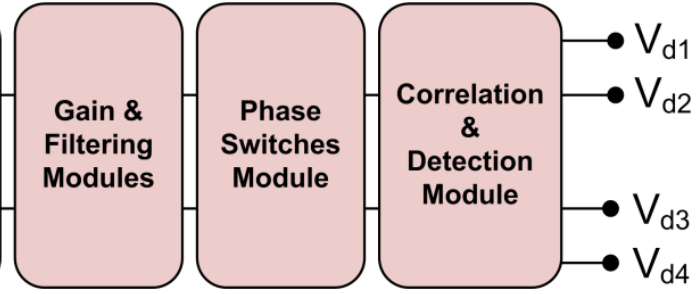

Back-End Module $(\mathrm{T}=300 \mathrm{~K})$

Fig. 20 Simplified schematic of the receiver for the cryogenic test

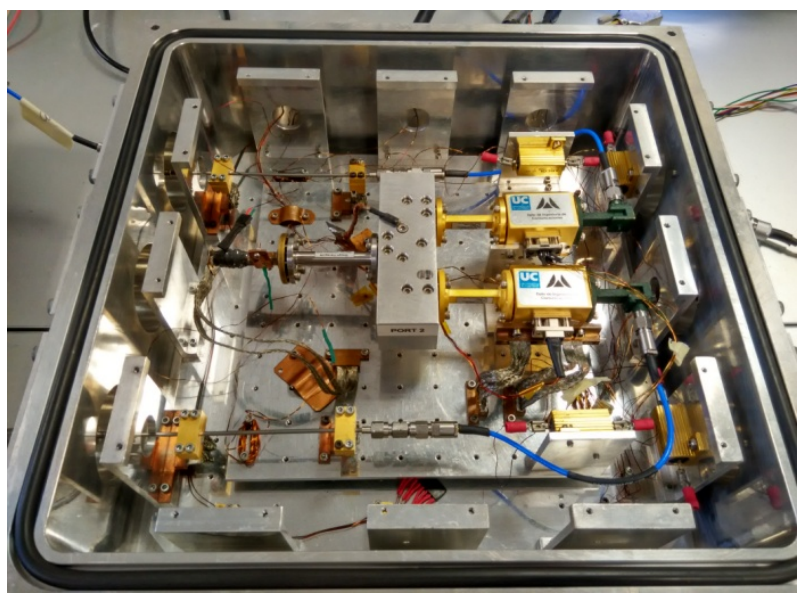

Fig. 21 Assembly of the front-end part for the test inside the cryostat

The assembly shown in Fig. 21 is connected to a Back-End Module at room temperature, integrated in a board as shown in Fig. 17. The output detected voltages are analysed in terms of noise temperatures in the receiver. Therefore, the four idealized detected outputs (the maximum, the minimum and the two mid-voltages signals corresponding to each phase state in an ideal receiver performance as listed in Table 2) are proportional to the receiver equivalent noise temperature and the noise introduced by the waveguide termination as follows:

$$
\begin{gathered}
V_{\max }=\alpha \cdot\left(\frac{T_{\text {receiver }}}{2}+\frac{T_{W G-\text { Term }}}{2}\right) \\
V_{\min }=\alpha \cdot\left(\frac{T_{\text {receiver }}}{2}\right) \\
V_{\text {med } 1}=V_{\text {med } 2}=\alpha \cdot\left(\frac{T_{\text {receiver }}}{2}+\frac{T_{W G-\text { Term }}}{4}\right)
\end{gathered}
$$


where $\alpha$ is the responsivity of the receiver, $T_{\text {receiver }}$ is the receiver equivalent noise temperature and $T_{W G-T e r m}$ is the noise temperature of the waveguide termination at the input of the receiver. These voltages are obtained at the paired outputs of each hybrid $V_{d 1}, V_{d 2}$ or $V_{d 3}, V_{d 4}$ depending on the phase state of the receiver. On the other hand, the responsivity, $\alpha$, is also slightly different for each state, mainly due to the different insertion loss value of the Phase Switches Module.

Calculating the total voltage $V_{\text {total }}$ as the addition of the four detected voltages for any phase state, its value is expressed as

$$
V_{\text {total }}=V_{d 1}+V_{d 2}+V_{d 3}+V_{d 4}=\alpha \cdot\left(2 \cdot T_{\text {receiver }}+T_{W G-T e r m}\right)
$$

which includes the total noise temperature of the waveguide termination at the input and twice the noise of the receiver due to the two branches contribution in the Front-End Module. Applying two different temperatures to the waveguide termination ( $T_{W G-T e r m 1}$ and $T_{W G-T e r m 2}$ with $T_{W G-T e r m 2}>T_{W G-T e r m 1}$ ), the receiver equivalent noise temperature $\left(T_{\text {receiver }}\right)$ can be obtained using the Y-factor method from the equation:

$$
T_{\text {receiver }}=\frac{1}{2} \cdot \frac{T_{W G-T e r m 2}-Y \cdot T_{W G-T e r m 1}}{Y-1}
$$

where the Y-factor is calculated with the detected voltages as follows:

$$
Y=\frac{\left.V_{\text {total }}\right|_{T_{W G-\text { Term 2 }}}}{\left.V_{\text {total }}\right|_{T_{W G-T e r m 1}}}
$$

This test is performed acquiring the four output detected voltages during a period of time equal to $1 \mathrm{~s}$ with a sampling rate of 1000 samples/s, setting four different fixed termination waveguide temperatures and a switching frequency of $16 \mathrm{~Hz}$. The waveguide termination temperature is fixed at $15 \mathrm{~K}, 50 \mathrm{~K}, 70 \mathrm{~K}$ and $88 \mathrm{~K}$ consecutively. $V_{\text {total }}$ is calculated, using Eq. 23, from the detected voltages for each sample and for all the input temperatures. The obtained values of $V_{\text {total }}$ for the 16 states are depicted in Fig. 22(a). Regarding the different responsivity for each state, the average value of $V_{\text {total }}$ for two of the states versus the waveguide termination temperature is calculated and depicted in Fig. 22(b), showing a linear behaviour for the receiver. The chosen states are marked as $E_{H}$ and $E_{L}$ in Fig. 22, which corresponds to the states with the highest and the lowest responsivity respectively.

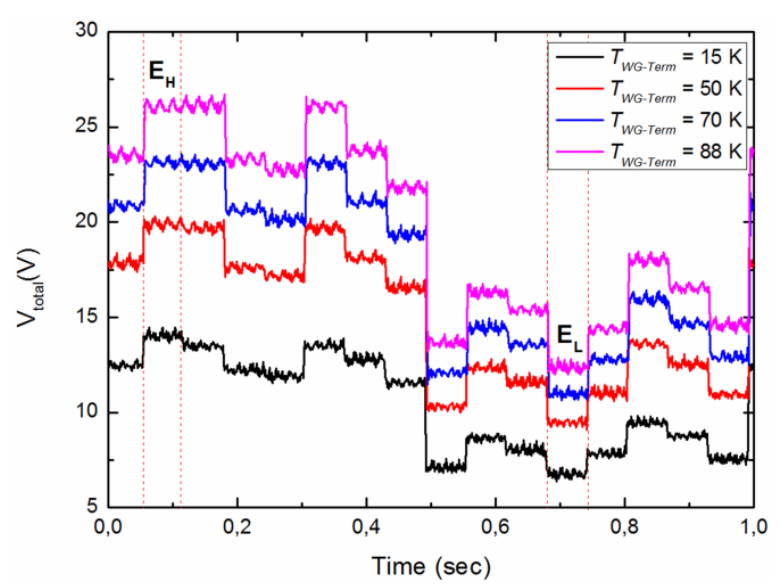

(a)

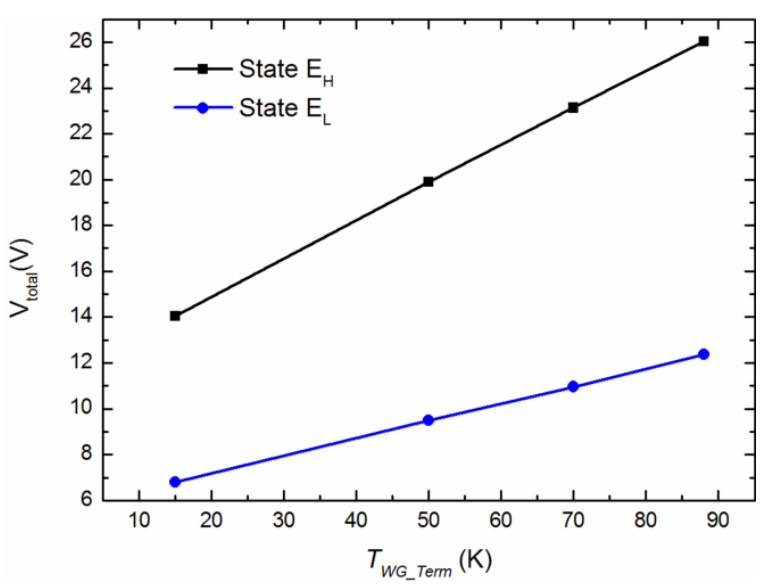

(b)

Fig. 22 Output detected voltages $V_{\text {total }}$ for different termination waveguide temperatures. (a) Acquisition time of $1 \mathrm{~s}$ showing the 16 phase states, $E_{H}$ indicates the state with the highest responsivity and $E_{L}$ the state with the lowest responsivity (b) Average $V_{\text {total }}$ versus temperature for states $\mathrm{E}_{\mathrm{H}}$ and $\mathrm{E}_{\mathrm{L}}$ 
The receiver noise temperature does not depend on the insertion loss of the Phase Switches Module since the LNAs in the Front-End Module together with those in the Back-End Module provide a gain of more than $70 \mathrm{~dB}$. Therefore, for any phase state with two different waveguide termination temperatures, the receiver equivalent noise temperature can be calculated according to Eq. 24 and Eq. 25. Using the values of $V_{\text {total }}$ at $T_{W G-}$ Term of $50 \mathrm{~K}$ and $88 \mathrm{~K}$ for each state in Fig. 22(a), the average Y-factor obtained for the 16 states, applying Eq. 25 is 1.168, which corresponds to $34.5 \mathrm{~K}$ receiver noise temperature calculated with Eq. 24. This value has the contribution to the noise of the SS waveguide and the in-phase waveguide power divider, both cooled down to $15 \mathrm{~K}$, which show together around $1.2 \mathrm{~dB}$ insertion loss. This insertion loss is slightly higher than the feed system composed of the polarizer and the OMT, which have, as a whole, $0.5 \mathrm{~dB}$ insertion loss, while the receiver noise temperature is around $27 \mathrm{~K}$.

Two long-term data acquisitions of the detected voltages with the waveguide termination at $88 \mathrm{~K}$ are performed. Both acquisitions last 5 minutes with 3200 samples/s but with a different phase configuration. The first one is configured with a constant phase state, while in the second one a switching rate of $16 \mathrm{~Hz}$ is applied. For a fixed phase state, the receiver is working as a total power radiometer and the output detected voltage spectrum, shown in Fig. 23(a), is obtained applying a fast-Fourier transform (FFT) to the maximum detected output voltage. The maximum detected voltage is $V_{d 1}=V_{\max }$ for the phase state chosen, which shows a DC output voltage measured of $4.7 \mathrm{~V}$. The effective bandwidth (Kraus 1986) of the receiver is calculated from the total power radiometer sensitivity expression as follows:

$$
\frac{\Delta T}{T_{s y s}}=\frac{\Delta V}{V}=\frac{1}{\sqrt{\beta \cdot \tau}}
$$

where $\Delta T$ is the sensitivity of the radiometer, $T_{\text {sys }}$ the system equivalent noise temperature, $\Delta V$ is the root-meansquare (RMS) value of the output voltage noise, $V$ is the DC output detected voltage, $\beta$ is the effective bandwidth and $\tau$ is the integration time. The obtained effective bandwidth for the receiver without switching is 12.3 GHz using Eq. 26, with $\mathrm{V}=4.7 \mathrm{~V}$, integration time $0.5 \mathrm{~s}$ (Tiuri 1964), and the level of $\Delta V$ in RMS is determined by taking the average value in a section of white noise well away from the $1 / \mathrm{f}$ region of the low frequency spectrum in Fig. 23(a), which gives $6 \cdot 10^{-5} V_{R M S} / \sqrt{H z}$.

In order to demonstrate an improvement in $1 / \mathrm{f}$ knee frequency of the output power spectrum for switching operation with respect to the unswitched one, the FFT of the time series $\left(\left.V_{\min }\right|_{E 0}+\left.V_{\max }\right|_{E 180}\right)-\left(\left.V_{\min }\right|_{E 180}+\left.V_{\max }\right|_{E 0}\right)$ is calculated, where $\left.V_{\min }\right|_{E 0}$ and $\left.V_{\max }\right|_{E 0}$ are the minimum and maximum detected voltages for one phase state (E0) and $\left.V_{\min }\right|_{E 180}$ and $\left.V_{\max }\right|_{E 180}$ are the minimum and maximum detected voltages for a phase state (E180) with $180^{\circ}$ phase difference with respect to E0. The time series is obtained using the acquired data during 5 minutes with a switching frequency of $16 \mathrm{~Hz}$, and applying the FFT. Hence, the obtained output power spectrum is calculated and depicted in Fig. 23(b). These spectra show a 1/f knee frequency of around $2 \mathrm{~Hz}$ when the phase state is switched, while a value above $10 \mathrm{~Hz}$ when no switching operation applied. This performance is expected to be improved once the polarimeter is installed in the telescope since a fast switching frequency of $16 \mathrm{kHz}$ will be used and all the components of the full receiver will be included in the test, using a more complex acquisition set-up. The 1/f noise contamination on the astronomical observations is critical for the final scientific objectives, since it degrades the quality of the acquired data increasing the effective rms noise and the uncertainty in the power spectrum at low multipole values (Seiffert 
2002, Janssen 1996, Maino 1999, Maino 2002). In CMB observations the statistical properties of the cosmic signal are degraded with the $1 / \mathrm{f}$ noise, increasing the overall noise level, and causing undesired strips in the final sky maps (Jansen 1996).

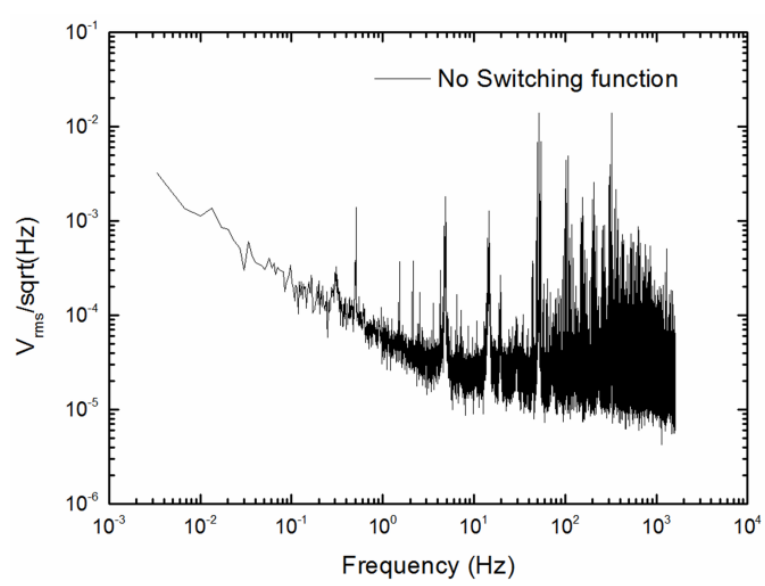

(a)

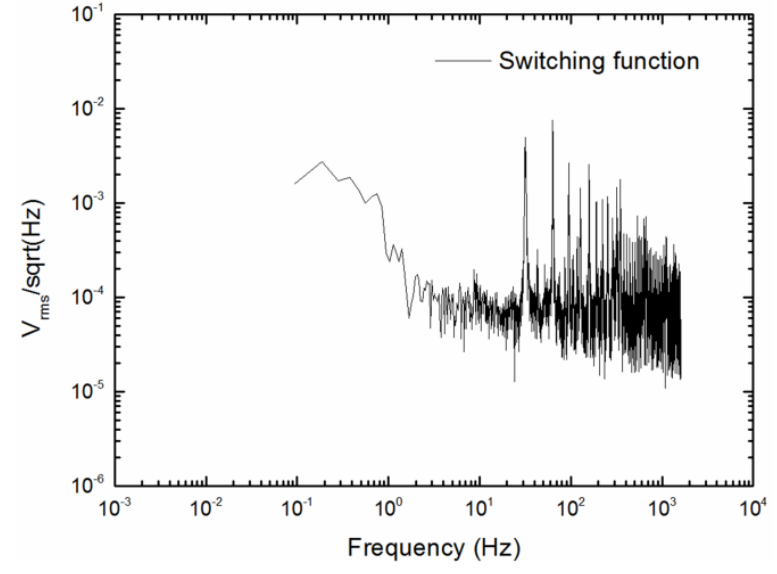

(b)

Fig. 23 Output power spectrum. (a) Unswitched configuration. (b) Switched configuration

\section{CONCLUSION}

The analysis, design and characterization of the FGI receiver for the QUIJOTE instrument working in the 35 to $47 \mathrm{GHz}$ frequency band have been presented. The theoretical analysis of the receiver is described in terms of the output voltages, which enable the calculation of the Stokes parameters, and noise performance. The different subsystems which compose the full receiver have been described, showing outstanding performances in a bandwidth greater than a 30\% at Q-band using waveguide and hybrid planar technologies. The receiver functionality has been demonstrated performing measurements at both room and cryogenic temperatures. A full switching capability test has been performed at room temperature, obtaining significant results in terms of the calculation of the Stokes parameters ( $I, Q$ and $U$ ) for a broadband noise-like x-axis linearly polarized input signal, with good agreement between the values of $I$ and $Q$ parameters and low error in the value of the $U$ parameter. The analysis of the sensitivity of one pixel of the polarimeter has been estimated under cryogenic operation of the feed part in the Front End Module, obtaining an equivalent receiver noise temperature of around $35 \mathrm{~K}$ and an improved performance when switching function is applied to the system reducing the $1 / \mathrm{f}$ knee frequency of the output power spectrum.

\section{REFERENCES}

1. Richards, P. L.: Cosmic Microwave Background experiments — past, present and future. 32nd International Conference on Infrared and Millimeter Waves and 15th International Conference on Terahertz Electronics (2007). https://doi.org/10.1109/ICIMW.2007.4516378

2. Aja, B., Artal, E., de la Fuente, L., Pascual, J. P., Mediavilla, A., Roddis, N., Kettle, D., Winder, W. F., Pradell L., de Paco, P.: Very low-noise differential radiometer at $30 \mathrm{GHz}$ for the PLANCK LFI. IEEE Trans. on Microw. Theory and Tech. (2005). https://doi.org/10.1109/TMTT.2005.848815

3. Bersanelli, M. et al.: Planck pre-launch status: Design and description of the Low Frequency Instrument. Astronomy \& Astrophysics. (2010). https://doi.org/10.1051/0004-6361/200912853

4. Aghanim, N. et al.: Planck 2013 results. III. LFI systematic uncertainties. Astronomy \& Astrophysics. (2014). https://doi.org/10.1051/0004-6361/201321574

5. National Aeronautics and Space Administration. Goddard Space Flight Center. Legacy Archive for Microwave Background Data Analysis. https://lambda.gsfc.nasa.gov/product/ (2017) 
6. Rubiño-Martin, J. A., Rebolo, R., Tucci, M., Génova-Santos, R., Hildebrandt, S. R., Hoyland, R., Herreros, J. M., Gómez-Renasco, F., López, C., Martínez-González, E., Vielva, P., Herranz, D., Casas, F. J., Artal, E., Aja, B., de la Fuente, L., Cano, J. L., Villa, E., Mediavilla, A., Pascual, J. P., Piccirillo, L., Maffei, B., Pisano, G., Watson, R. A., Davis, R., Davies, R., Battye, R., Saunders, R., Grainge, K., Scott, P., Hobson, M., Lasenby, A., Murga, G., Gómez, C., Gómez, A., Arino, J., Sanquirce, R., Pan, J., Vizcarguenaga A., Etxeita, B.: Highlights of Spanish Astrophysics V, Astrophysics and Space Science Proceedings, Part 3 pp. 127-135. Springer, Berlin (2010).

7. López-Caniego, M. et al.: The QUIJOTE CMB Experiment: status and first results with the multi-frequency instrument. Instrumentation and Methods for Astrophysics, Conference Proceedings Rencontres du Vietnam 2013: Cosmology in the Planck Era (2014). https://arxiv.org/abs/1401.4690.

8. Villa, E., Cano, J. L., Cagigas, J., Ortiz, D., Casas, F. J., Pérez, A. R., Aja, B., Terán, J. V., de la Fuente, L., Artal, E., Hoyland R., Mediavilla, A.: The thirty gigahertz instrument receiver for the Q-U-I Joint Tenerife experiment: Concept and experimental results. Rev. Sci. Instrum. (2015). http://dx.doi.org/10.1063/1.4907015.

9. Villa, E.: Wideband Microwave Circuits for RadioAstronomy Applications. Ph. D. thesis, Universidad de Cantabria. https://www.educacion.gob.es/teseo/mostrarRef.do?ref=1123302 (2014).

10. Villa, E., Cagigas, J., Aja, B., de la Fuente, L., Artal, E.: Q-band 4-state phase shifter in planar technology: Circuit design and performance analysis. Rev. Sci. Instrum. (2016). http://dx.doi.org/10.1063/1.4963322.

11. Collet, E.: Field Guide to Polarization. SPIE Press, Bellingham (2005).

12. Otoshi, T. Y.: Noise Temperature Theory and Applications for Deep Space Communications Antenna Systems. Artech House, Norwood (2008).

13. Cano, J. L., Villa, E., Aja, B., de la Fuente, L., Artal, E., Watson, R., Blackhurst, E., Edgley, J., Baines, C.: The Ka-band receiver for the QUIJOTE experiment. 6th European Microwave Integrated Circuits Conference, 620-623 (2011). http://ieeexplore.ieee.org/document/6102820/.

14. Tribak, A., Mediavilla, A., Cano, J. L., Boussouis, M., Cepero, K.: Ultra-broadband low axial ratio corrugated quad-ridge polarizer. 39th European Microwave Conference (2009). https://doi.org/10.23919/EUMC.2009.5295927.

15. Cano, J. L., Tribak, A., Hoyland, R., Mediavilla, A., Artal, E.: Full band waveguide turnstile junction orthomode transducer with phase matched outputs. Int. Jrnl. RF and Microw. CAE (2010). http://dx.doi.org/10.1002/mmce.20437.

16. Aja, B., Seelmann-Eggebert, M., Bruch, D., Leuther, A., Massler, H., Baldischweiler, B., Schlechtweg, M., Gallego-Puyol, J. D., López-Fernández, I., Díez-González, C., Malo-Gómez, I., Villa, E., Artal, E.: 4-12- and 25-34-GHz Cryogenic mHEMT MMIC Low-Noise Amplifiers. IEEE Trans. on Microw. Theory and Tech. (2012). https://doi.org/10.1109/TMTT.2012.2221735.

17. Zhou, T. F., Wang, Z. G., Huan, W., Xu, R. M.: Design of microwave wave gain equalizer using microstrip shorted SIR. International Conference on Microwave and Millimeter Wave Technology (2012). https://doi.org/10.1109/ICMMT.2012.6230399.

18. Terán, J. V.: Low nose receivers for Millimetre-wave bands radio astronomy. Ph. D. thesis, Universidad de Cantabria. https://www.educacion.gob.es/teseo/mostrarRef.do?ref=1448502 (2017).

19. Kraus, J. D.: Radio Astronomy. Cygnus-Quasar Books, Ohio (1986).

20. Tiuri, M. E.: Radio astronomy receivers. IEEE Transactions on Antennas and Propagation (1964). https://doi.org/10.1109/TAP.1964.1138345.

21. Seiffert, M., Mennella, A., Burigana, C., Mandolesi, N., Bersanelli, M., Meinhold, P., Lubin, P.: 1/f Noise and other Sistematic Effects in the Planck-LFI Radiometers. Astronomy \& Astrophysics (2002). https://doi.org/10.1051/0004-6361:20020880

22. Janssen, M. A., Scott, D., White, M., Seiffert, M. D., Lawrence, C. R., Górski, K. M., Dragovan, M., Gaier, T., Ganga, K., Gulkis, S., Lange, A. E., Levin, S. M., Lubin, P. M., Meinhold, P., Readhead, A. C. S., Richards, P. L., Ruhl, J. E.: Direct Imaging of the CMB from Space. Cornell University Library - Astrophysics (1996). https://arxiv.org/abs/astro-ph/9602009

23. Maino, D., Burigana, C., Maltoni, M., Wandelt, B. D., Górski, K. M., Malaspina, M., Bersanelli, M., Mandolesi, N., Banday, A. J., Hivon, E.: The Planck-LFI instrument: Analysis of the 1/f noise and implications for the scanning strategy. Astronomy \& Astrophysics (1999). https://doi.org/10.1051/aas:1999429

24. Maino, D., Burigana, C., Górski, K. M., Mandolesi, N., Bersanelli, M.: Removing the 1/f noise in cosmic microwave background anisotropy observations. Astronomy \& Astrophysics (2002). https://doi.org/10.1051/0004-6361:20020242 